\title{
Prevalence of primary bacterial co-infections among patients with COVID-19 in Brunei Darussalam
}

Aieman Bashir a Muhammad Syafig Abdullah b Natalie Raimiza Momin b Pui Lin Chong b Rosmonaliza Asli, b Babu Mani lvan and Vui Heng Chong ${ }^{a, b}$

Correspondence to Vui Heng Chong (email: vuiheng.chong@moh.gov.bn)

Objective: Bacterial co-infections in cases of coronavirus disease 2019 (COVID-19) can lead to less favourable outcomes. The aim of this study was to determine the prevalence of primary bacterial co-infections among patients with COVID-19 in Brunei Darussalam.

Methods: Seventy-one of 180 patients admitted to the National Isolation Centre between 9 March 2020 and 4 February 2021 were screened for primary bacterial co-infection (infection occurring $\leq 48 \mathrm{~h}$ from admission). We compared patients with a primary bacterial co-infection to those without.

Results: Of the 71 screened patients, 8 (11.2\%) had a primary bacterial co-infection (sputum 37.5\% [6/16], blood 2.8\% [1/36], urine $1.7 \%[1 / 60]$ ), for a period prevalence rate of $4.4 \%$ (respiratory tract infection $3.3 \%$ [6/180], bloodstream $0.6 \%$ [1/180], urine 0.6\% [1/180]) among all COVID-19 patients. Older age, presence of comorbidity, symptoms at admission (fever, dyspnoea, nausea/vomiting), abnormal chest X-ray (CXR) and more severe COVID-19 $(P<0.05)$ were associated with primary bacterial co-infection. Primary bacterial co-infection was also associated with development of secondary infection and death (all $P<0.05$ ). Only one patient with primary bacterial co-infection died (methicillin-sensitive Staphylococcus aureus septicaemia and multiorgan failure).

Conclusions: Our study showed that primary bacterial co-infection affected $4.4 \%$ of patients with COVID-19 in Brunei Darussalam. Older age, presence of comorbidity, symptoms and abnormal CXR at admission and more severe disease were associated with a primary bacterial co-infection. Lower respiratory tract infection was the most common co-infection.

$\mathrm{C}$ oronavirus disease 2019 (COVID-19), caused by severe acute respiratory syndrome coronavirus 2 (SARS-CoV-2), is associated with significant morbidity and mortality, especially among susceptible populations such as the elderly and those with comorbidities. ${ }^{1}$ Co-infections have been reported in COVID-19 patients and can lead to less favourable outcomes. $^{2-5}$ To date, most studies have reported overall co-infection rates and have not distinguished between primary and later co-infections. Primary or early co-infection is defined as any infection occurring simultaneously or detected soon after admission, usually within 48 or 72 h. Any co-infection occurring after this period is regarded as secondary co-infection.

Brunei Darussalam reported its first COVID-19 case on 9 March 2020, and, as of 4 February 2021, had recorded 180 cases. The country remains at WHO level
2 of transmission, ${ }^{6}$ with sporadic imported cases and no local infection since 6 May 2020. All patients diagnosed with COVID-19, regardless of symptoms, are admitted to the National Isolation Centre (NIC) for isolation and treatment. We report our nationwide experience in screening for primary bacterial co-infection among patients treated for COVID-19 in Brunei Darussalam.

\section{METHODS}

In this descriptive study, data were retrospectively retrieved from a prospectively maintained database. This Excel ${ }^{\circledR}$ database was created at the start of the COVID-19 outbreak to monitor patients and for situational management and analysis. The information collected includes demographic data, comorbidities, symptoms at admission and during hospitalization, investigations, treatment and outcomes.

\footnotetext{
Department of Medicine, Pengiran Muda Mahkota Pengiran Muda Haji Al-Muhtadee Billah Hospital, Tutong, Brunei Darussalam.

Department of Medicine, Raja Isteri Pengiran Anak Saleha Hospital, Bandar Seri Begawan, Brunei Darussalam.

Published: 8 September 2021

doi: 10.5365/wpsar.2021.12.3.856
} 


\section{COVID-19 management and categorization}

In Brunei Darussalam, all patients who test positive for SARS-CoV-2 by reverse transcription polymerase chain reaction (RT-PCR) are admitted to the NIC (designated hospital for COVID-19 for the whole country) for isolation and treatment.

Patients were categorized as symptomatic if they had symptoms at admission, asymptomatic if they had no symptoms during their illness and pre-symptomatic if they were asymptomatic at admission but developed symptoms during hospitalization. Management was based on the treatment protocol described in our previous publication. ${ }^{7}$

Patients were categorized into three levels of disease severity: mild (asymptomatic or mild disease, i.e. with none of the features listed under the moderate and severe categories), moderate (fever with respiratory tract symptoms and pneumonia on imaging) and severe (respiratory rate $>30 / \mathrm{min}$, oxygen saturation $\leq 93 \%$ at rest, $\mathrm{PaO} 2 / \mathrm{FiO} 2 \leq 300 \mathrm{~mm} \mathrm{Hg}$ and progression of lung lesions $>50 \%$ within $24-48 \mathrm{~h}$ ). Patients categorized as having mild COVID-19 were managed in the main isolation wards, and those with moderate or severe COVID-19 were managed in the high-dependency or intensive-care setting.

\section{Screening for primary bacterial co-infection}

Primary bacterial co-infection was defined as infection diagnosed within $48 \mathrm{~h}$ of admission. Screening for primary bacterial co-infection was carried out according to our management protocol. ${ }^{7}$ At admission, a detailed history and clinical examinations were carried out for all patients, and all had routine investigations (blood and chest X-ray [CXR]). Patients with fever, rigor, cough, shortness of breath, dysuria, diarrhoea, abnormal investigational findings (leukocytosis, elevated inflammatory markers [C-reactive protein], elevated procalcitonin) and abnormal CXR were screened for primary co-infection by collection of sputum, urine and stool for Gram staining, microscopy and culture, and blood for culture.

\section{Management of co-infections}

As our management protocol included a 5-day course of oseltamivir to cover influenza $A$ and $B$, we did not screen for influenza viruses. We also did not screen for other common respiratory viral pathogens, as no specific treatment is available. After investigations for primary bacterial co-infection, empiric antimicrobials were administered when indicated and later adjusted according to culture sensitivity patterns for those who screened positive. Patients who developed or continued to have symptoms $48 \mathrm{~h}$ after admission were screened for secondary coinfections, defined as infection occurring more than $48 \mathrm{~h}$ after admission. Clinical management was guided by the investigational results.

\section{Data analyses}

Data were anonymized and extracted from our Excel ${ }^{\circledR}$ database for analysis with IBM SPSS version 23.0. In our management protocol, only patients who had symptoms or abnormal findings of investigations at admission were screened for primary bacterial co-infections. Patients who were not screened were included in the group with no primary bacterial co-infections for analyses. We assessed factors associated with positivity for primary bacterial co-infection (demographic characteristics, symptoms, investigations, disease severity) and outcomes (screening and positivity for secondary co-infection, death) using the Mann-Whitney test for continuous variables and the Fisher exact test for categorical variables. A $P$ value of $<0.05$ was considered statistically significant.

\section{RESULTS}

As of 4 February 2021, 180 patients (mean age, 35.3 \pm 16.7 years; males, $62.2 \%$ ) had been admitted to the NIC with COVID-19. Among them, 28.9\% $(n=52)$ had comorbidities, including hypertension (13.3\%), dyslipidaemia (13.9\%), diabetes mellitus (5.6\%), pulmonary disease $(4.4 \%)$ and cardiac disease (3.3\%). Overall, $56.1 \%(n=101)$ were symptomatic, $6.7 \%(n=12)$ were pre-symptomatic and $37.2 \%(n=67)$ were asymptomatic. Common symptoms included cough (33.9\%), fever $(23.3 \%)$ and rhinorrhoea (20.0\%). An abnormal CXR at admission was noted in $15.3 \%$, and the cycle threshold value was $27.2 \pm 6.3$. The majority of cases were categorized as mild, with $11.7 \%$ categorized as moderate and $2.7 \%$ as severe (Table 1 ).

Primary bacterial co-infection was screened in 71 (39.4\%) patients. Of these, $80.3 \%$ (57 patients) were screened within $24 \mathrm{~h}$ and $19.7 \%$ (14 patients) $24-48 \mathrm{~h}$ after admission. Of the 71 patients screened, $8(11.2 \%)$ had a primary bacterial co-infection, usually in the lower 
Table 1. Characteristics and outcomes of patients

\begin{tabular}{|c|c|c|c|c|}
\hline Variable & $\begin{array}{l}\text { All patients } \\
(N=180) \\
n(\%)\end{array}$ & $\begin{array}{l}\text { Positive for primary } \\
\text { co-infection }(n=8)\end{array}$ & $\begin{array}{l}\text { Negative for primary } \\
\text { co-infection }(n=172)\end{array}$ & $P$ \\
\hline \multicolumn{5}{|l|}{ Demography and characteristics } \\
\hline Mean age (years) & $35.3 \pm 16.7$ & $50.6 \pm 12.6$ & $34.6 \pm 16.5$ & \multirow{2}{*}{0.007} \\
\hline Median (range (years)) & $33(0.58-72)$ & $53(29-64)$ & $32(0.58-72)$ & \\
\hline \multicolumn{5}{|l|}{ Gender } \\
\hline Male & $112(62.2)$ & $6(75.0)$ & $106(61.6)$ & \multirow[t]{2}{*}{0.446} \\
\hline Female & $68(37.8)$ & $2(25.0$ & $66(38.4)$ & \\
\hline Comorbidity (yes) & $52(28.9)$ & $5(62.5)$ & $47(27.3)$ & 0.032 \\
\hline Hypertension & $24(13.3)$ & $4(50.0)$ & $20(11.6)$ & 0.002 \\
\hline Diabetes & $10(5.6)$ & $2(25.0)$ & $8(4.7)$ & 0.014 \\
\hline Dyslipidaemia & 25 (13.9) & $5(62.5)$ & 20 (11.6) & $<0.001$ \\
\hline Cardiac disease & $6(4.3)$ & $1(12.5)$ & $5(2.9)$ & 0.14 \\
\hline Pulmonary disease & $8(4.4)$ & $1(12.5)$ & $7(4.1)$ & 0.259 \\
\hline Symptoms at admission (yes) & $101(56.1)$ & $6(75.0)$ & $95(55.2)$ & 0.271 \\
\hline Fever & $42(23.3)$ & $5(62.5)$ & $37(21.5)$ & 0.007 \\
\hline Cough & $61(33.9)$ & $4(50.0)$ & $57(33.1)$ & 0.325 \\
\hline Rhinorrhoea & $36(20.0)$ & $2(25.0)$ & $34(19.8)$ & 0.718 \\
\hline Diarrhoea & $9(5.0)$ & $1(12.5)$ & $8(4.7)$ & 0.319 \\
\hline Anosmia & $2(1.1)$ & $0(0.0)$ & $2(1.2)$ & 0.759 \\
\hline Myalgia & $16(8.9)$ & $1(12.5)$ & $15(8.7)$ & 0.714 \\
\hline Sore throat & $12(6.7)$ & $0(0.0)$ & $12(7.0)$ & 0.439 \\
\hline Headache & $13(7.2)$ & $0(0.0)$ & $13(7.6)$ & 0.419 \\
\hline Nausea/vomiting & $3(1.7)$ & $1(12.5)$ & $2(1.2)$ & 0.014 \\
\hline Dyspnoea & $4(2.1)$ & $3(37.5)$ & $1(0.6)$ & $<0.001$ \\
\hline \multicolumn{5}{|l|}{ Admission investigations } \\
\hline \multicolumn{5}{|l|}{ Chest X-ray* } \\
\hline Abnormal & $26(15.3)$ & $4(50.0)$ & $22(13.6)$ & 0.005 \\
\hline \multicolumn{5}{|l|}{ Investigations } \\
\hline Cycle threshold & $27.2 \pm 6.3$ & $25.3 \pm 6.8$ & $27.2 \pm 6.3$ & 0.336 \\
\hline Albumin & $39.4 \pm 3.9$ & $38.3 \pm 2.9$ & $39.4 \pm 3.97$ & 0.314 \\
\hline Neutrophil & $3.8 \pm 2.2$ & $4.10 \pm 2.93$ & $3.82 \pm 2.16$ & 0.94 \\
\hline Lymphocyte & $2.0 \pm 0.8$ & $1.61 \pm 0.66$ & $1.97 \pm 0.77$ & 0.294 \\
\hline Neutrophil-lymphocyte ratio & $2.39 \pm 2.39$ & $3.17 \pm 2.65$ & $2.35 \pm 2.38$ & 0.429 \\
\hline \multicolumn{5}{|l|}{ Outcomes } \\
\hline \multicolumn{5}{|l|}{ Disease category } \\
\hline Mild & $154(85.6)$ & $4(50.0)$ & $150(87.2)$ & \multirow{3}{*}{0.012 for trend } \\
\hline Moderate & $21(11.7)$ & $3(37.5)$ & $18(10.5)$ & \\
\hline Severe/critical & $5(2.7)$ & $1(12.5)$ & $4(2.3)$ & \\
\hline Screened for secondary co-infection & $20(11.1)$ & $3(37.5)$ & $17(9.9)$ & 0.015 \\
\hline Developed secondary co-infection & $10(5.6)$ & $2(25.0)$ & $8(4.7)$ & 0.014 \\
\hline Death (mortality rate) & $3(1.7)$ & $1(12.5)$ & $2(1.2)$ & 0.014 \\
\hline
\end{tabular}

*Ten patients did not undergo chest $\mathrm{X}$-ray. 
Table 2. Details of screening and result for primary bacterial co-infections

\begin{tabular}{|c|c|c|c|c|c|}
\hline Sample & $\begin{array}{c}n(\%) \\
(N=180)\end{array}$ & $\begin{array}{c}\text { Positivity rate } \\
n(\%)\end{array}$ & Case no. & Organism/s & Outcomes \\
\hline Blood & $36(20.0)$ & $1(2.8)$ & 1 & Methicillin-sensitive Staphylococcus aureus & Died of septicaemia/ARDS \\
\hline Urine & $60(33.3)$ & $1(1.7)$ & 2 & Streptococcus group B & Treated and alive \\
\hline \multirow{6}{*}{ Sputum } & \multirow{6}{*}{$16(8.9)$} & \multirow{6}{*}{$6(37.5)$} & 3 & Rothia mucilaginosa/Enterobacter gergoviae & \multirow{6}{*}{ All treated and alive } \\
\hline & & & 4 & Klebsiella pneumoniae/MRSA & \\
\hline & & & 5 & Haemophilus influenzae & \\
\hline & & & 6 & Streptococcus pneumoniae & \\
\hline & & & 7 & Klebsiella pneumoniae & \\
\hline & & & 8 & Enterobacter aerogenes & \\
\hline Stool & $2(1.1)$ & $0(0.0)$ & - & Negative & - \\
\hline
\end{tabular}

ARDS: acute respiratory distress syndrome; MRSA: methicillin-resistant Staphylococcus aureus.

respiratory tract, for a period prevalence of $4.4 \%$ among all COVID-19 patients. Most of the positive samples were sputum (6/16 patients [37.5\%]), followed by blood (1/36 patients [2.8\%]) and urine (1/60 patients [1.7\%]). Stool assessment showed no bacteria. The bacteria isolated consisted of Klebsiella species (2 patients), Streptococcus spp. (2 patients), methicillin-resistant Staphylococcus species (2 patients), Enterobacter species (2 patients), Rothia mucilaginosa (1 patient) and Haemophilus influenzae (1 patient). Two organisms were isolated from two patients (cases 3 and 4 in Table 2).

One patient (case 4) with methicillin-resistant Staphylococcus aureus (MRSA) isolated from sputum had a history of clinic visits, and screening showed colonization only in the nose. The patient (case 2) with group B Streptococcus isolated in urine reported no urinary symptoms but had fever, myalgia and nausea at admission. He was treated for urinary infection with a course of co-amoxiclav. Blood and sputum cultures from this patient were negative.

Patients with primary bacterial co-infection tended to be older, had comorbidities (especially hypertension, diabetes mellitus and dyslipidaemia), reported symptoms at admission (fever, nausea/vomiting, dyspnoea), had abnormal CXR and were categorized as having moderate or severe disease (Table 1). These patients were also more likely than those without primary bacterial coinfection to be screened for ( $37.5 \%$ vs. $9.9 \%, P=0.015)$ and develop secondary co-infection $(25 \%$ vs. $4.7 \%$, $P=0.014)$ and to die $(12.5 \%$ vs. $0.6 \%, P=0.014)$.

One of the patients with primary bacterial coinfection died 16 days after admission due to MRSA septicaemia. This patient presented late (5 days after the onset of dyspnoea), was symptomatic at admission and deteriorated rapidly. The CXR was consistent with pneumonia. Sputum Gram staining was positive for Gram-positive cocci, but no organism was isolated. All the other patients with primary bacterial co-infection recovered.

\section{DISCUSSION}

We assessed the period prevalence of primary bacterial co-infection, factors and outcomes among patients with COVID-19 in Brunei Darussalam. The period prevalence was $4.4 \%$, consistent with the results of other published studies. In one study in Spain, 37 primary co-infections were reported in 31 (of 989) patients admitted with COVID-19, a prevalence of 3.1\%; 30 were bacterial coinfections (in 25 patients) and 7 were viral co-infections. Two patients among those with bacterial co-infection were infected with two different organisms. ${ }^{8}$ A study in the United States of America found that 2.8\% of COVID-19 patients had primary respiratory tract co-infection and $1.1 \%$ had bacteraemia. The authors screened $80(14.7 \%)$ of 542 patients with COVID-19 for primary bacterial respiratory co-infection, of whom 15 $(2.8 \%)$ were treated as having true primary respiratory co-infections, defined as any infection identified within $72 \mathrm{~h}$ of admission. ${ }^{9}$ Although we did not screen patients for viral co-infection, the rate has been reported to be $1.5-3.0 \% .^{2,10,11}$ Respiratory syncytial virus and influenza $A$ are the two most common, with detection rates of $16.9 \%$ and $15.5 \%$, respectively. ${ }^{2}$

Most of the co-infections in our study were of the lower respiratory tract. As COVID-19 is mainly a 
respiratory illness, primary and secondary bacterial co-infections can occur, as in other respiratory viral illnesses. ${ }^{2,3,8,9}$ Therefore, it is not surprising that we found that the respiratory tract was the most common site of bacterial co-infection and that sputum samples had the highest proportion of positive results.

Overall, a wide spectrum of organisms has been found in COVID-19 co-infections, with some patients having more than one co-infection. In a study in China, 24 types of respiratory pathogen were found in respiratory samples from patients with COVID-19, of whom $94.2 \%$ were co-infected with one or more pathogens. Common bacterial pathogens reported included $S$. pneumoniae, K. pneumoniae and $H$. influenzae. ${ }^{12}$ In our study, several organisms were isolated from two patients: $R$. mucilaginosa and $E$. gergoviae (case 3 ) and MRSA and $K$. pneumoniae (case 4), which are uncommon organisms. The reported spectrum of organisms in nosocomial co-infections among hospitalized COVID-19 patients is similar to those encountered in clinical practice among non-COVID-19 patients. ${ }^{13,14}$

Although few bacterial and viral co-infections have been identified in COVID-19 patients, vigilance must be maintained, with screening for both primary and secondary co-infections, as they have been associated with less favourable outcomes. ${ }^{9}$ In our study, COVID-19 patients with primary bacterial co-infection were older, had comorbidities, presented with fever, dyspnoea and nausea/vomiting, had an abnormal CXR at admission and were categorized as having moderate or severe disease. COVID-19 patients with primary bacterial co-infection were also more likely to be screened for and develop secondary infection and were more likely to die. One systematic review and meta-analysis showed that the odds of death were higher for patients with co-infections and superinfection than for those with only SARS-CoV-2 infection (odds ratio $=3.31 ; 95 \%$ confidence interval, 1.82-5.99). ${ }^{15}$

Strengths and limitations of our study should be considered when interpreting the findings. The main strength of our study is that it included all COVID-19 cases in the country and may therefore be applicable to countries or regions with similar demographics, healthcare infrastructure and management of the COVID-19 pandemic. An important limitation is the small numbers of COVID-19 patients and of primary bacterial coinfections, as small samples make the results liable to inherent errors in statistical analysis. Another limitation is the evolving nature of our management protocol in the earlier phase of the outbreak. Apart from normal symptom screening and investigations, some patients also underwent opportunistic screening of easily obtained samples, such as urine. Furthermore, not all our patients were screened, and patients with mild or asymptomatic primary bacterial co-infection might have been missed. The proportion is, however, likely small. Importantly, our study represents a real-world situation in which screening is conducted mainly when indicated.

In conclusion, we have shown that primary bacterial co-infection, mostly in the respiratory tract, has affected $4.4 \%$ of COVID-19 patients in Brunei Darussalam. The rate of primary bacterial co-infection was higher among older patients and those with comorbidities, symptoms at admission (particularly fever, nausea/vomiting and dyspnoea), an abnormal CXR and moderate or severe disease. Primary bacterial co-infection was also associated with secondary co-infection and death. Routine blood and urine screening for bacterial co-infection is not indicated and should be guided by clinical and laboratory indicators; however, primary co-infections should be identified early and treated appropriately to prevent complications.

\section{Acknowledgements}

None

\section{Conflicts of interest}

None for all authors

\section{Ethics}

This study was conducted in accordance with the Declaration of Helsinki 2013 and data used for analyses were anonymized.

\section{Funding}

None 


\section{References}

1. Jordan RE, Adab P, Cheng KK. Covid-19: risk factors for severe disease and death. BMJ. 2020;368:m1198. doi:10.1136/bmj.m1198 pmid:32217618

2. Lansbury L, Lim B, Baskaran V, Lim WS. Co-infections in people with COVID-19: a systematic review and meta-analysis. Infect. 2020;81(2):266-75. doi:10.1016/j.jinf.2020.05.046 pmid:32473235

3. Rawson TM, Moore LSP, Zhu N, Ranganathan N, Skolimowska K, Gilchrist $\mathrm{M}$ et al. Bacterial and fungal co-infection in individuals with coronavirus: A rapid review to support COVID-19 antimicrobial prescribing. Clin Infect Dis. 2020;71(9):2459-68. doi:10.1093/cid/ ciaa530 pmid:32358954

4. Cox MJ, Loman N, Bogaert D, O'Grady J. Co-infections: potentially lethal and unexplored in COVID-19. Lancet Microbe. 2020;1(1):e11. doi:10.1016/S2666-5247(20)30009-4 pmid:32835323

5. Bardi T, Pintado V, Gomez-Rojo M, Escudero-Sanchez R, Azzam Lopez A, Diez-Remesal Y et al. Nosocomial infections associated to COVID-19 in the intensive care unit: clinical characteristics and outcome. Eur J Clin Microbiol Infect Dis. 2021;40(3):495-502. doi:10.1007/s10096-020-04142-w pmid:33389263

6. Coronavirus disease 2019 (COVID-19). Situation report 72. 1 April 2020. Geneva: World Health Organization; 2020. Available from: https://www.who.int/emergencies/diseases/novel-coronavirus-2019/situation-reports, accessed 10 June 2021.

7. Chong VH, Chong PL, Metussin D, Asli R, Momin RN, Mani Bl et al Conduction abnormalities in hydroxychloroquine add on therapy to lopinavir/ritonavir in COVID-19. J Med Virol. 2020;92(11):23224. doi:10.1002/jmv.26004 pmid:32401368

8. Garcia-Vidal C, Sanjuan G, Moreno-García E, Puerta-Alcalde P, Garcia-Pouton N, Chumbita M et al. Incidence of co-infections and superinfections in hospitalized patients with COVID-19: a retrospective cohort study. Clin Microbiol Infect. 2021;27(1):83-8. doi:10.1016/j.cmi.2020.07.041 pmid:32745596
9. Lardaro $T$, Wang AZ, Bucca A, Croft A, Glober N, Holt DB et al. Characteristics of COVID-19 patients with bacterial co-infection admitted to the hospital from the emergency department in a large regional healthcare system. J Med Virol. 2021;93(5):2883-9. doi:10.1002/jmv.26795 pmid:33448423

10. Burrel S, Hausfater P, Dres M, Pourcher V, Luyt CE, Teyssou E et al. Co-infection of SARS-CoV-2 with other respiratory viruses and performance of lower respiratory tract samples for the diagnosis of COVID-19. Int J Infect Dis. 2021;102:10-3. doi:10.1016/j. ijid.2020.10.040 pmid:33115679

11. Peci A, Tran V, Guthrie JL, Li Y, Nelson P, Schwartz KL et al. Prevalence of co-infections with respiratory viruses in individuals investigated for SARS-CoV-2 in Ontario, Canada. Viruses. 2021;13:130. doi:10.3390/v13010130 pmid:33477649

12. Zhu X, Ge $Y$, Wu T, Zhao K, Chen $Y, W u$ B et al. Co-infection with respiratory pathogens among COVID-2019 cases. Virus Res. 2020;285:198005. doi:10.1016/j.virusres.2020.198005 pmid:32408156

13. Song JH, Huh K, Chung DR. Community-acquired pneumonia in the Asia-Pacific region. Semin Respir Crit Care Med. 2016;37(6):83954. doi:10.1055/s-0036-1592075 pmid:27960208

14. Torres A, Blasi F, Peetermans WE, Viegi G, Welte T. The aetiology and antibiotic management of community-acquired pneumonia in adults in Europe: a literature review. Eur J Clin Microbiol Infect Dis. 2014;33(7):1065-79. doi:10.1007/s10096-014-2067-1 pmid:24532008

15. Musuuza JS, Watson L, Parmasad V, Putman-Buehler N, Christensen L, Safdar N. Prevalence and outcomes of co-infection and superinfection with SARS-CoV-2 and other pathogens: A systematic review and meta-analysis. PLoS One. 2021;16(5):e0251170. doi:10.1371/journal.pone.0251170 pmid:33956882 